
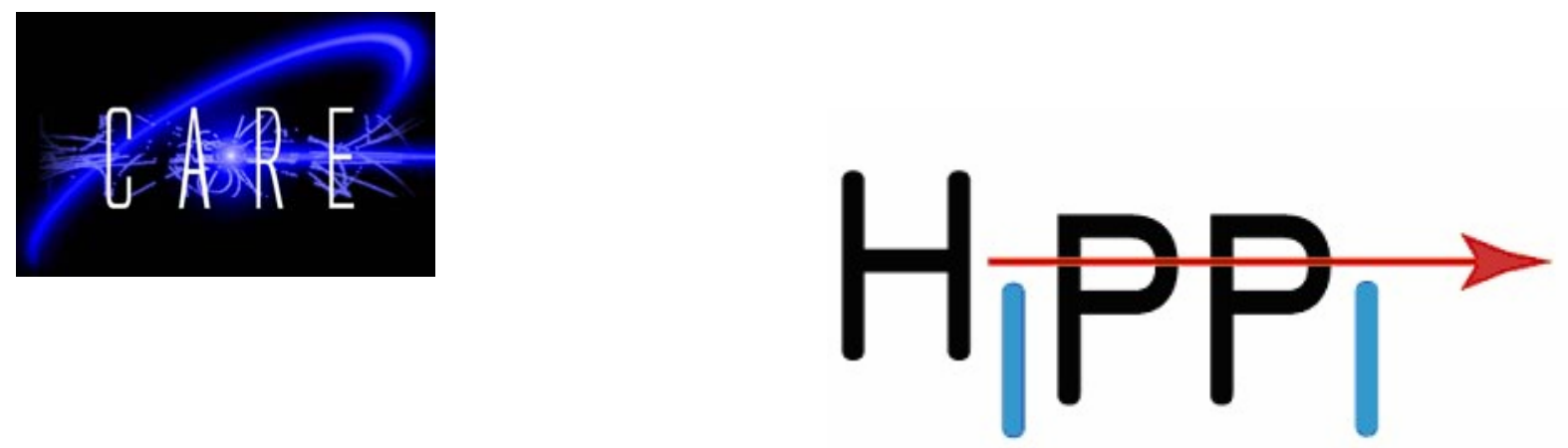

The SPL at CERN

R. Garoby

CERN, Geneva - Switzerland

\begin{abstract}
The Superconducting Proton Linac (SPL) is a multi-GeV, multi-MW linear proton machine which represents a very interesting option for the future of CERN. Operating at $50 \mathrm{~Hz}$, it will serve both as a high performance injector for the complex of high energy accelerators, replacing the present PS booster, and as a high power proton driver for other physics applications like the generation of muons and neutrinos and/or radio-active ions. The potential of the SPL is presently being scrutinized and compared to other alternatives. A path of evolution is considered, beginning with the realization of a $3 \mathrm{MeV}$ test facility, followed by the building of the $160 \mathrm{MeV}$ front end of the SPL and finishing with the construction of the complete linac. The status of these investigations and the progress towards the SPL are summarized in this paper.
\end{abstract}

Contribution to the Workshop ICFA-HB2004, Bensheim, Germany

Work supported by the European Community-Research Infrastructure Activity under the FP6 "Structuring the European Research Area" programme (CARE, contract number RII3-CT2003-506395). 


\title{
The SPL At CERN ${ }^{1}$
}

\author{
R. Garoby
}

CERN, Geneva - Switzerland

\begin{abstract}
The Superconducting Proton Linac (SPL) is a multi-GeV, multi-MW linear proton machine which represents a very interesting option for the future of CERN. Operating at $50 \mathrm{~Hz}$, it will serve both as a high performance injector for the complex of high energy accelerators, replacing the present PS booster, and as a high power proton driver for other physics applications like the generation of muons and neutrinos and/or radio-active ions. The potential of the SPL is presently being scrutinized and compared to other alternatives. A path of evolution is considered, beginning with the realization of a $3 \mathrm{MeV}$ test facility, followed by the building of the $160 \mathrm{MeV}$ front end of the SPL and finishing with the construction of the complete linac. The status of these investigations and the progress towards the SPL are summarized in this paper.
\end{abstract}

Keywords: Superconducting RF, proton, Linac. PACS: $29.17+\mathrm{w}$, 29.27.Fh, 41.75.Ak, 41.75.Cn

\section{INTRODUCTION}

CERN is presently completing the construction of the Large Hadron Collider (LHC), the world largest particle accelerator. Once this machine will be operational, the other accelerators, which serve as injectors, will deserve improvement or replacement because of their respectable age (30-40 years) and because of the new needs of physics in the next decade. The Superconducting Proton Linac (SPL) is a proposed successor to the present Linac2 and PS Booster (PSB) that would provide a higher performance beam for high energy physics uses, including the LHC, and enough beam power for a neutrino facility and for a second generation ISOLtype radio-active ion beam facility.

\section{THE SPL}

Since the publication of the first Conceptual Design Report [1], the physics requests have evolved and the SPL characteristics and its design are being revised accordingly. The nominal mean beam power is kept at $4 \mathrm{MW}$, but the beam energy is increased up to $3.5 \mathrm{GeV}$ which is optimum for a neutrino super-beam [2]. The beam current during the pulse is increased up to $30-40 \mathrm{~mA}$ (the final value depends upon the outcome of on-going studies), which reduces the duration of the beam pulse, allows the use of pulsed klystrons and eases the design of the following synchrotron. These parameters are being used for the preparation of a second Conceptual Design Report to be published in 2005. They are summarized in Table 1.

TABLE 1. Main SPL characteristics

\begin{tabular}{lcc} 
TABLE 1. Main SPL characteristics & CDR-1 & CDR-2 \\
& $(\mathbf{2 0 0 0 )}$ & $\begin{array}{c}\text { (2005) } \\
\text { (200 }\end{array}$ \\
\hline Ion species & \multicolumn{3}{c}{$\mathrm{H}^{-}$} \\
Kinetic energy [GeV] & 2.2 & \multicolumn{2}{c}{3.5} \\
Mean beam power [MW] & \multicolumn{2}{c}{4} \\
Beam current during pulse [mA] & 13 & \multicolumn{2}{c}{$30-40$} \\
Pulse repetition rate [Hz] & \multicolumn{2}{c}{50} \\
Pulse duration [ms] & 2.8 & $0.76-0.57$ \\
Bunch frequency [MHz] & \multicolumn{2}{c}{352.2} \\
Normalised rms transverse & \multicolumn{2}{c}{0.4} \\
emittances [ $\pi$ mm mrad] & \multicolumn{2}{c}{0.3} \\
Longit. rms emittance $[\pi$ deg MeV] & \multicolumn{2}{c}{0.3} \\
\hline
\end{tabular}

\section{High Energy Superconducting Linac}

The initial design was severely constrained by the characteristics of the LEP RF equipment that was planned to be re-used in the superconducting part of

\footnotetext{
${ }^{1}$ Work supported by the European Community-Research Infrastructure Activity under the FP6 "Structuring the European Research Area" programme (CARE, contract number RII3-CT-2003-506395)
} 
the linac (total peak power and accelerating gradient). The new design will be better optimized and exploit LEP RF equipment only in the low energy part where it is fully justified. Harmonics of the LEP RF frequency (352.2 MHz) will be used in the rest of the accelerator. A higher RF peak power will be available from new pulsed klystrons, and higher accelerating gradients will be provided by the smaller accelerating structures, less prone to mechanical vibrations.

\section{Low Energy - Normal Conducting Injector (“Linac4")}

The low energy part of the SPL, called Linac4, is planned to be first used as a replacement of Linac2, the present PSB injector. It is based on normal conducting accelerating structures and its main characteristics in the two possible modes of operation are given in Table 2.

\section{TABLE 2. Main characteristics of Linac4}

\begin{tabular}{|c|c|c|}
\hline & $\begin{array}{c}\text { Phase 1 } \\
\text { (PSB) }\end{array}$ & $\begin{array}{c}\text { Phase } 2 \\
\text { (SPL) }\end{array}$ \\
\hline Kinetic energy [MeV] & \multicolumn{2}{|c|}{160} \\
\hline Pulse repetition rate [Hz] & 2 & 50 \\
\hline Source current [mA] & \multicolumn{2}{|c|}{$50-65$} \\
\hline RFQ current [mA] & \multicolumn{2}{|c|}{$40-55$} \\
\hline Current after chopper [mA] & \multicolumn{2}{|c|}{$30-40$} \\
\hline Maximum pulse duration [ms] & 0.5 & 2 \\
\hline Maximum beam duty cycle [\%] & 0.1 & 10 \\
\hline $\begin{array}{l}\text { Normalised rms transverse } \\
\text { emittances }[\pi \mathrm{mm} \text { mrad] }\end{array}$ & \multicolumn{2}{|c|}{0.33} \\
\hline Longit. rms emittance $[\pi$ deg $\mathrm{MeV}]$ & \multicolumn{2}{|c|}{0.24} \\
\hline
\end{tabular}

Compared to the initial parameters, the energy is increased to $160 \mathrm{MeV}$ to double the intensity and the brightness of the beam delivered by the PSB [3]. The pulse length when driving the SPL is conservatively set at $2 \mathrm{~ms}$ to provide more beam power at $3.5 \mathrm{GeV}$ or the same beam power at a lower energy. A new design has been worked out [4] in which the RF frequency is doubled above $90 \mathrm{MeV}$ (704 MHz) to minimize length and optimize performance of the SPL. The corresponding layout is shown in Figure 1.

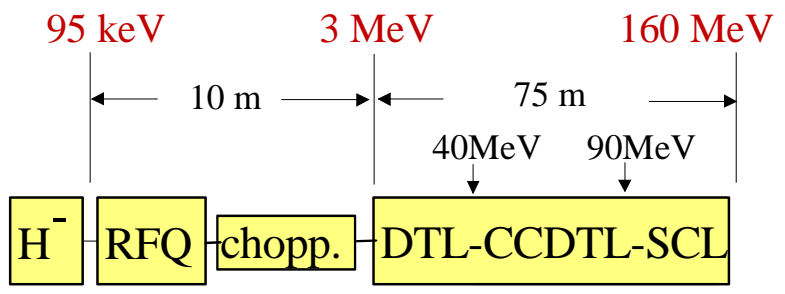

FIGURE 1. Schematic Linac4 layout.
Table 3 shows the main characteristics of the successive sections. The front end is based on the RFQ designed and built by CEA and IN2P3 in France in the IPHI project [5], which will be transferred to CERN at the end of a series of tests at Saclay in 2007 [6]. Designed for CW operation, this RFQ will only be used at CERN at a much lower duty cycle $(<10 \%)$.

At $3 \mathrm{MeV}$, the chopper line generates the beam time structure required for optimum longitudinal capture in a synchrotron. The $3.6 \mathrm{~m}$ long line is based on a FODO focusing structure and contains two $500 \mathrm{~mm}$ long deflecting structures located inside quadrupoles, a beam dump, three bunching cavities as well as diagnostics and focusing elements. It is also equipped with movable scrapers and a collimation system to remove halo particles coming from the source, the RFQ and the line itself. An important argument in the choice of $3 \mathrm{MeV}$ for the energy of the chopper line and the transition from RFQ to DTL, is that it is considered as the highest value where the cross-section for nuclear reactions in $\mathrm{Cu}$ are still small enough that equipment activation is negligible.

TABLE 3. SPL normal conducting section (Linac4)

\begin{tabular}{lccccc}
\hline & $\begin{array}{c}\text { Output } \\
\text { energy } \\
{[\mathbf{M e V}]}\end{array}$ & $\begin{array}{c}\text { Nb. of } \\
\text { cavities } \\
\text { (tanks) }\end{array}$ & $\begin{array}{c}\text { Peak RF } \\
\text { power } \\
{[\mathbf{M W}]}\end{array}$ & $\begin{array}{c}\text { Nb. of } \\
\text { klystrons }\end{array}$ & $\begin{array}{c}\text { Length } \\
{[\mathbf{m}]}\end{array}$ \\
\hline LEBT & 0.095 & - & - & - & 2 \\
RFQ & 3 & 1 & 0.9 & 1 & 6 \\
Chopper & 3 & 3 & 0.1 & - & 3.7 \\
line & & & & & \\
DTL & 40.2 & 3 & 4.8 & 5 & 16.7 \\
CCDTL & 90 & 27 & 5.6 & 6 & 30.1 \\
SCL & 160 & 20 & 13.8 & 5 & 27.8 \\
Total & & 54 & 25.2 & 17 & 86.3 \\
\hline
\end{tabular}

An Alvarez Drift Tube Linac (DTL) follows the RFQ, up to an energy of $40 \mathrm{MeV}$. This section is made of 3 tanks, the first one fed by one klystron, and the others by two klystrons each. Tank 1 is equipped with Permanent Magnet Quadrupoles (PMQ), while the other tanks have conventional electromagnetic quadrupoles (EMQ). Simulations show that the quadrupoles in the chopper line allow matching to the fixed gradient focusing channel of Tank 1 for a wide range of beam currents. FFDD focusing has been preferred for all the DTL [4].

Above $40 \mathrm{MeV}$ the focusing periods can be longer than $\beta \lambda$, and alternative structures can be adopted, with quadrupoles outside of the drift tubes. For Linac4, a particular Cell-Coupled Drift Tube Linac (CCDTL) design at $352 \mathrm{MHz}$ has been selected [7]. This structure is made of short 3-gap tanks connected by bridge couplers. The quadrupoles placed between tanks are mechanically independent of the RF 
structures, and can be easily aligned. Each klystron feeds a module made of 5 or 4 tanks via a single input coupler.

Beyond approximately $90 \mathrm{MeV}$, the shunt impedance of 0 -mode DTL-like structures starts to decrease drastically. Therefore a Side-Coupled Linac (SCL) design at $704 \mathrm{MHz}$ has been preferred for the energy range up to $160 \mathrm{MeV}$. This section is made of 20 tanks with 11 accelerating cells each, connected into 5 modules of 4 tanks, each fed by a $4 \mathrm{MW}$ klystron. Cell-to-cell coupling is $3 \%$, and tanks are connected by 3-cell bridge couplers.

Out of the 17 klystrons needed in Linac4, 12 operate at $352 \mathrm{MHz}$ and will come from the LEP inventory and 5 are new $704 \mathrm{MHz}$ devices.

To transport the beam from Linac 4 to the PSB, a $200 \mathrm{~m}$ long transfer line will be built, equipped with 2 RF cavities and 27 quadrupoles.

\section{Status Of Developments}

The realization of the $3 \mathrm{MeV}$ RFQ is the subject of an agreement between CEA, CERN and IN2P3, by which the IPHI device designed by the French institutions will finally be made available at CERN before the end of 2007. The front-end of Linac4, based on this RFQ, is presently under construction. It will first serve as a $3 \mathrm{MeV}$ test place (see Figure 2) during the year 2008 .

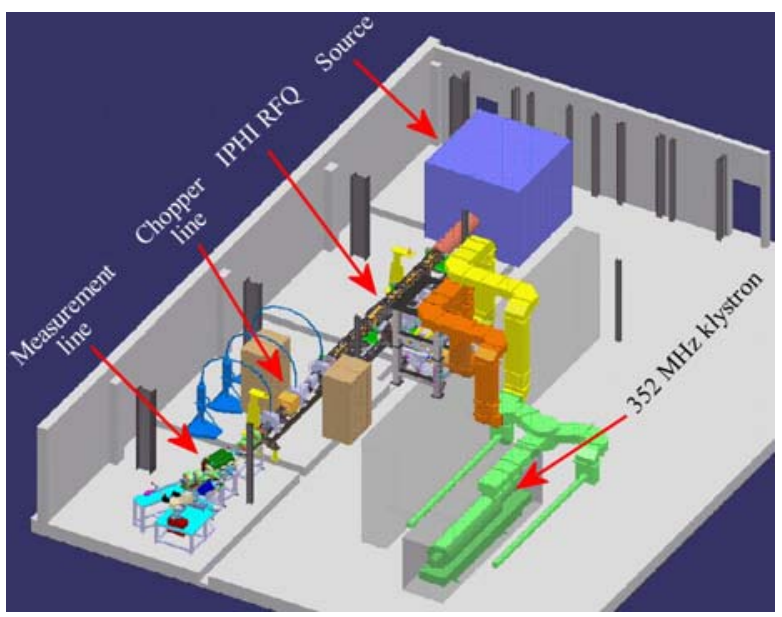

FIGURE 2. $3 \mathrm{D}$ view of the $3 \mathrm{MeV}$ test place.

With the support of the European Union, the recently approved HIPPI (High Intensity Proton Pulsed Injectors) Joint Research Activity (JRA) [8] covers a significant fraction of the R\&D programme for Linac4.
This JRA coordinates the efforts of 9 European Laboratories in the development of the technologies for the next generation of European high-intensity linac facilities over the period 2004-2008, addressing the subjects of normal- and super-conducting accelerating structures, chopping and codes for high intensity beam simulation. For hardware, prototypes of the most promising alternatives will be built and compared. For beam simulation, benchmarking will be made during extensive measurements campaigns on UNILAC at GSI and on the $3 \mathrm{MeV}$ test place at CERN, using specially developed beam instrumentation.

This programme is complemented by three projects funded by the International Science and Technology Center (ISTC- Moscow). They are aimed at the design and construction, in Russia, of prototypes of normalconducting accelerating structures for Linac4. In the first one, which involves BINP (Novosibirsk) and VNIITF (Snezhinsk), a high-power CCDTL prototype and an SCL cold model will be built before the end of 2005. In the second one, concerning ITEP (Moscow) and VNIIEF (Sarov), a prototype Alvarez DTL tank will be realized before the end of 2006. The third one, lead by IHEP (Protvino) and VNIIEF (Sarov), is focused on the realization of a low-cost alternative to the Alvarez DTL ("DTL-RFQ") before the end of 2006.

The foreseen planning of all these activities is shown in Figure 3. The CERN management has already endorsed the date of the end of 2006 to decide upon the construction of Linac4. The date for the decision concerning the SPL is governed by the need to have the accelerator available when the LHC will be shutdown for upgrade in $2014 \pm 1$ year.

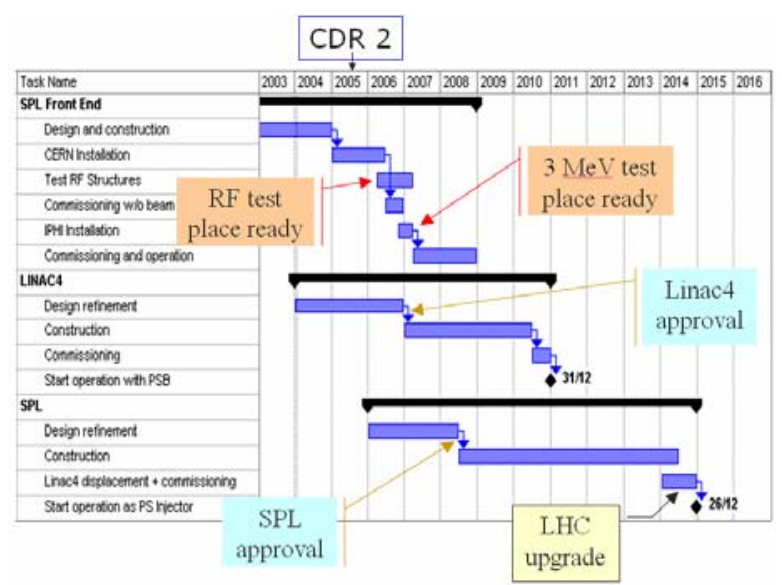

FIGURE 3. Integrated planning for proton linacs. 


\section{PERSPECTIVE FOR FUTURE PROTON ACCELERATORS AT CERN}

To operate and make use of all the potential for physics of the LHC, the complex of proton accelerators at CERN, which serve as injectors, will deserve an extensive consolidation and improvement programme during the years 2010-2015. The upgrade of the LHC itself has to be prepared during these years, and it is likely to have major consequences on the lower energy machines. Moreover, during most of the time, the LHC is in coasting mode at $7 \mathrm{TeV}$, so that the beams available from the injectors can be used for other purposes. Multiple physics communities are interested and preparing their plans for the next decade.
A first approach to these questions has been made by the High Intensity Proton (HIP) working group inside the $\mathrm{AB}$ department, taking into account the actual commitments of CERN and the requests expressed by the physics communities interested in neutrinos and radio-active ion beams. A number of recommendations have resulted for the short, medium and long term [9]. The construction of Linac4 is supported for its medium term benefits to the LHC and ISOLDE. For the longer term, the HIP working group has tried to qualify the relative benefits of various alternatives. After refinement during workshops with physicists in 2004 [10, 11], the comparative table has evolved to the version exposed in Table 4. Although the ideal scenario depends strongly upon the new physics programmes that will be approved, the SPL has clearly a broad potential that justifies the continuation of the development efforts.

TABLE 4. Possible long term improvements to the CERN complex of proton accelerators

("RCS"=Rapide Cycling Synchrotron, "HEP"=High Energy Phyics, “mMW"=multi-MW, “SC"=Superconducting, “CNGS”=CERN Neutrinos to Gran Sasso, “RIB”=Radio-active Ion Beams).

\begin{tabular}{|c|c|c|c|c|c|c|}
\hline \multirow[b]{2}{*}{$\begin{array}{c}\text { Present } \\
\text { accelerator }\end{array}$} & \multirow[b]{2}{*}{$\begin{array}{c}\text { Replacement } \\
\text { accelerator }\end{array}$} & \multirow[b]{2}{*}{$\begin{array}{c}\text { Main } \\
\text { Improvements }\end{array}$} & \multicolumn{4}{|c|}{ Interest for } \\
\hline & & & LHC upgrade & $\begin{array}{c}\text { v physics beyond } \\
\text { CNGS }\end{array}$ & $\begin{array}{c}\text { RIB } \\
\text { beyond } \\
\text { ISOLDE }\end{array}$ & $\begin{array}{c}\text { Physics } \\
\text { with kaons } \\
\text { and } \mu\end{array}$ \\
\hline Linac2 & Linac4 & $\begin{array}{c}50 \rightarrow 160 \mathrm{MeV} \\
\mathrm{H}^{+} \rightarrow \mathrm{H}^{-}\end{array}$ & $\begin{array}{c}+ \\
\text { Filling time } \downarrow \\
\text { PS brightness } \times 1.3\end{array}$ & $\begin{array}{c}\text { None } \\
\text { (if alone) }\end{array}$ & $\begin{array}{l}\text { None } \\
\text { (if alone) }\end{array}$ & $\begin{array}{l}\text { None } \\
\text { (if alone) }\end{array}$ \\
\hline \multirow{3}{*}{ PSB } & $\begin{array}{c}2.2 \mathrm{GeV} \text { RCS } \\
\text { for HEP }\end{array}$ & $\begin{array}{c}1.4 \rightarrow 2.2 \mathrm{GeV} \\
0.01 \rightarrow 0.25 \mathrm{MW}\end{array}$ & $\begin{array}{c}+ \\
\text { Filling time } \downarrow \\
\text { PS brightness } \uparrow\end{array}$ & $\begin{array}{c}\text { None } \\
\text { (if alone) }\end{array}$ & + & $\begin{array}{c}\text { None } \\
\text { (if alone) }\end{array}$ \\
\hline & $\begin{array}{c}2.2 \mathrm{GeV} \text { mMW } \\
\text { RCS’s }\end{array}$ & $\begin{array}{l}1.4 \rightarrow 2.2 \mathrm{GeV} \\
0.01 \rightarrow 4 \mathrm{MW}\end{array}$ & $\begin{array}{c}+ \\
\text { Filling time } \downarrow \\
\text { PS brightness } \uparrow\end{array}$ & $\begin{array}{c}+++ \text { for } \\
\text { super-beam, beta- } \\
\text { beam and } v \text { factory }\end{array}$ & $\begin{array}{c}+ \\
\text { (too short } \\
\text { beam pulse) }\end{array}$ & $\begin{array}{l}\text { None } \\
\text { (if alone) }\end{array}$ \\
\hline & $\begin{array}{c}2.2 \mathrm{GeV} / 50 \mathrm{~Hz} \\
\mathrm{SPL}\end{array}$ & $\begin{array}{l}1.4 \rightarrow 2.2 \mathrm{GeV} \\
0.01 \rightarrow 4 \mathrm{MW}\end{array}$ & $\begin{array}{c}+ \\
\text { Filling time } \downarrow \\
\text { PS brightness } \uparrow \uparrow\end{array}$ & $\begin{array}{c}+++ \text { for } \\
\text { super-beam, beta- } \\
\text { beam and } v \text { factory }\end{array}$ & +++ & $\begin{array}{l}\text { None } \\
\text { (if alone) }\end{array}$ \\
\hline \multirow{2}{*}{ PS } & $\begin{array}{c}50 \mathrm{GeV} \text { SC PS } \\
\text { for HEP }\end{array}$ & $26 \rightarrow 50 \mathrm{GeV}$ & $\begin{array}{c}++ \\
\text { Filling time } \downarrow \\
\text { PS brightness } \uparrow\end{array}$ & $\begin{array}{l}\text { None } \\
\text { (if alone) }\end{array}$ & None & + \\
\hline & $\begin{array}{c}50 \mathrm{GeV} / 5 \mathrm{~Hz} \\
\mathrm{RCS}\end{array}$ & $\begin{array}{l}26 \rightarrow 50 \mathrm{GeV} \\
0.1 \rightarrow 4 \mathrm{MW}\end{array}$ & $\begin{array}{c}++ \\
\text { Filling time } \downarrow \downarrow \\
\text { PS brightness } \uparrow\end{array}$ & ++ & None & +++ \\
\hline SPS & $1 \mathrm{TeV}$ SC SPS & $0.45 \rightarrow 1 \mathrm{TeV}$ & $\begin{array}{c}+++ \\
\text { LHC injection } \uparrow \\
\text { SPS brightness } \uparrow\end{array}$ & ? & None & +++ \\
\hline
\end{tabular}

In September 2004, the physics committee in charge of the fixed target experiments at CERN (SPSC) had a special meeting to analyze the experiments proposed for the next decade and to make recommendations to the direction [12]. The potential for physics of the SPL has been presented, as well as the outcome of the HIP working group. In its preliminary recommendations, the SPSC made it clear that there is a need for a multi-MW proton source at CERN in the next decade. Considering the importance of physics with kaons and muons, the beam energy should exceed $\sim 20 \mathrm{GeV}$. Therefore the solutions based on Rapid Cycling Synchrotrons have to be investigated in more detail.

Early in 2005, the physics committee in charge of experiments with radio-active ion beams and of the 
"Neutrons-time-of-flight" facility (INTC) will hold a similar meeting and provide its own recommendations to the CERN direction.

Combined with the studies actively pursued in the E.U. supported networks "BENE" (for neutrino physics) and "H3" (for LHC upgrade) inside CARE ("Coordinated Accelerator Research in Europe"), these inputs will contribute to the preparation of the decisions about the future of CERN after LHC commissioning.

\section{ACKNOWLEDGMENTS}

The linac developments are the work of numerous contributors all over the world. Thanks to the support of the European Union, their number has noticeably increased in 2004 with the beginning of the HIPPI JRA [8]. Thanks to the ISTC funding, 5 Russian institutions are now providing efforts.

Close collaboration with physicists is essential for a proper optimization of the accelerator(s). I'd like to thank especially Profs. A. Blondel, V. Palladino and P. Butler for their pedagogy and patience.

In the present stressful context of construction of the LHC, the constructive attitude of the CERN management with respect to longer term perspectives augurs well for the future of our organization.

\section{REFERENCES}

1. M. Vretenar (editor), "Conceptual Design of the SPL, a High Power Superconducting $\mathrm{H}^{-}$Linac at CERN", CERN 2000-012.

2. Campagne, J.E., Cazes, A., "The $\vartheta_{13}$ and $\delta_{C P}$ sensitivities of the SPL-Frejus project revisited", LAL04-102 and submitted to Eur. Phys. J., C.

3. Martini, M. and Prior, C., "High-Intensity and HighDensity Charge-Exchange Injection Studies into the CERN PS Booster at Intermediate Energies”, CERNAB-2004-069 and Proceedings of EPAC2004, pp. 18911893.

4. Garoby, R., Gerigk, F., Hanke, K., Lombardi, A., Rossi, C., Vretenar, M., "Design of LINAC4, a New Injector for the CERN Booster", CERN-AB-2004-104 and Proceedings of LINAC2004 Conference, Lubeck, August 2004.

5. Beauvais, P.Y. et al., "Status on the Saclay High Intensity Proton Injector Project (IPHI)”, Proceedings of EPAC’00, Vienna, June 2000, pp. 283-285.
6. Garoby, R. et al., "The SPL Front-End: a $3 \mathrm{MeV}$ Test Stand at CERN”, CERN-AB-2004-102 and Proceedings of LINAC2004 Conference, Lubeck, August 2004.

7. Vretenar, M. et al., "Development of a $352 \mathrm{MHz}$ CellCoupled Drift Tube Linac Prototype”, CERN-AB-2004103 and Proceedings of LINAC2004 Conference, Lubeck, August 2004.

8. http://mgt-hippi.web.cern.ch/mgt-hippi/.

9. Benedikt, M., Cornelis, K., Garoby, R. Metral, E., Ruggiero, F. Vretenar, M. "Report of the High Intensity Protons Working Group”, CERN-AB-2004-022 OP/RF.

10. Physics with a multi-MW Proton Source [CERN (CH) May 2004]

11. High Intensity Frontier Workshop [Elba Island (I) - June 2004]

12. SPSC meeting [Villars (CH) - September 2004] 